Claremont Colleges

Scholarship@ Claremont

All HMC Faculty Publications and Research

HMC Faculty Scholarship

$10-1-2008$

\title{
Tiling Proofs of Recent Sum Identities Involving Pell Numbers
}

\author{
Arthur T. Benjamin \\ Harvey Mudd College \\ Sean S. Plott '08 \\ Harvey Mudd College \\ James A. Sellers \\ Pennsylvania State University - Main Campus
}

\section{Recommended Citation}

Benjamin, A.T., Plott, S.S., \& Sellers, J.A. (2008). Tiling proofs of recent sum identities involving Pell numbers. Annals of Combinatorics, 12(3): 271-278. DOI: 10.1007/s00026-008-0350-5.

This Article is brought to you for free and open access by the HMC Faculty Scholarship at Scholarship @ Claremont. It has been accepted for inclusion in All HMC Faculty Publications and Research by an authorized administrator of Scholarship @ Claremont. For more information, please contact scholarship@cuc.claremont.edu. 


\title{
Tiling Proofs of Recent Sum Identities Involving Pell Numbers
}

\author{
Arthur T. Benjamin \\ Department of Mathematics, Harvey Mudd College, \\ Claremont, CA 91711 \\ E-mail: benjamin@hmc.edu \\ Sean S. Plott \\ Department of Mathematics, Harvey Mudd College, \\ Claremont, CA 91711 \\ E-mail: splott@hmc.edu \\ and \\ James A. Sellers \\ Department of Mathematics, Penn State University, \\ University Park, PA 16802 \\ E-mail: sellersj@math.psu.edu \\ July 15, 2006 \\ Mathematics Subject Classification: 05A19
}

Abstract 
In a recent note, Santana and Diaz-Barrero proved a number of sum identities involving the well-known Pell numbers. Their proofs relied heavily on the Binet formula for the Pell numbers. Our goal in this note is to reconsider these identities from a purely combinatorial viewpoint. We provide bijective proofs for each of the results by interpreting the Pell numbers as enumerators of certain types of tilings. In turn, our proofs provide helpful insight for straightforward generalizations of a number of the identities.

Keywords: Pell numbers, combinatorial identities, tilings, NSW numbers 


\section{Motivation}

In a recent article, Santana and Diaz-Barrero [3] proved a number of sum identities involving the Pell numbers $P_{n}$ defined by $P_{0}=0, P_{1}=1$, and $P_{n}=2 P_{n-1}+P_{n-2}$ for $n \geq 2$. (See Sloane's Online Encyclopedia of Integer Sequences [4, A000129] for more details about the Pell numbers.) For example, Santana and Diaz-Barrero proved the following for all $n \geq 0$ :

Theorem 1. $\sum_{k=1}^{4 n+1} P_{k}$ is a perfect square.

Theorem 2.

$$
\sum_{r=0}^{n}\left(\begin{array}{c}
2 n+1 \\
2 r
\end{array}\right) 2^{r}=P_{2 n}+P_{2 n+1}
$$

\section{Theorem 3.}

$$
\sum_{r=0}^{n} \frac{2 n}{2 n-r}\left(\begin{array}{c}
2 n-r \\
r
\end{array}\right) 2^{2 n-2 r}=P_{2 n-1}+P_{2 n+1}
$$

and

$$
\sum_{r=0}^{n} \frac{2 n+1}{2 n+1-r}\left(\begin{array}{c}
2 n+1-r \\
r
\end{array}\right) 2^{2 n+1-2 r}=P_{2 n}+P_{2 n+2} .
$$

\section{Theorem 4.}

$$
P_{2 n+1} \text { divides } \sum_{k=0}^{2 n} P_{2 k+1}
$$

and

$$
P_{2 n} \text { divides } \sum_{k=1}^{2 n} P_{2 k-1} \text {. }
$$

The proofs of Theorems 14 provided by Santana and Diaz-Barrero, along with the proofs of three lemmas which also appear in [3], rely heavily on the Binet formula for $P_{n}$ along with various binomial coefficient identities. Our primary goal in this work is to view the above identities combinatorially, providing bijective arguments from the context of tilings as discussed in Benjamin and Quinn's recent book Proofs That Really Count [1]. In this way, we 
provide elegant proofs of the identities of Santana and Diaz-Barrero, proofs that can clearly be generalized to yield a variety of related sum identities, which are highlighted at the conclusion of this paper.

In order to provide combinatorial arguments for the identities above, we will consider the sequence of numbers denoted $p_{n}$ and defined as $p_{n}=P_{n+1}$ for $n \geq-1$. The motivation for considering $p_{n}$ is simple: $p_{n}$ counts the number of tilings of a board of length $n$ using white squares, black squares, and gray dominoes. Thus, for example, $p_{0}=1$ counts the empty tiling, $p_{1}=2$ because a board of length 1 can be covered (exactly) by either one white square or one black square. Similarly, $p_{2}=5$ since a board of length 2 , or a 2-board, can be covered by two white squares or two black squares or one white square and one black square or one gray domino. See Figure 1.
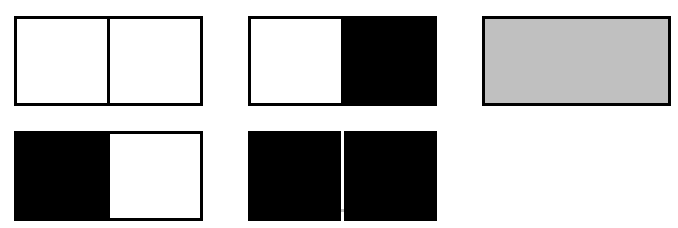

Figure 1: The tilings of a 2-board

It is within the context of such tilings that we restate and combinatorially prove Theorems 14 .

\section{Tiling Proofs}

We will consider Theorems 14 in reverse order, since our proofs of these results will consequently grow in complexity. 


\subsection{Theorem 4}

First, the two divisibility properties stated in this theorem can be combined into one statement. Moreover, this one divisibility property, when couched in the terms of $p_{n}$ rather than $P_{n}$, reads as follows: for all $n \geq 0$,

$$
p_{n} \text { divides } \sum_{k=0}^{n} p_{2 k}
$$

We begin our combinatorial proof of (1) by proving the following lemma:

Lemma 5. For all $n \geq 0$,

$$
p_{2 n+1}=2 \sum_{k=0}^{n} p_{2 k} .
$$

Proof. We begin by remembering that $p_{2 n+1}$ counts all tilings of a $(2 n+1)-$ board (i.e., a board of length $2 n+1$ with cells numbered from 1 to $2 n+1$ ). Note that each such tiling must contain at least one square since $2 n+1$ is odd. By considering the location of the last square, which must occur in an odd-numbered cell, we see that there are $2 p_{2 k}$ tilings whose last square occurs on cell $2 k+1(0 \leq k \leq n)$, where the factor of 2 indicates the color choice of this last square. Hence, the right-hand side of the equation also counts all tilings of a $(2 n+1)$-board.

With Lemma 5 in hand, we can now prove (1), as follows:

Theorem 6. For all $n \geq 0, p_{n}$ divides $p_{2 n+1}$. Moreover, $p_{n}$ divides $\sum_{k=0}^{n} p_{2 k}$.

Proof. We consider whether or not a tiling of length $2 n+1$ is breakable at cell $n$, i.e., if the $(2 n+1)$-tiling can be split into a tiling of length $n$ followed by a tiling of length $n+1$. There are $p_{n} p_{n+1}$ breakable tilings; the number of unbreakable tilings (with a domino covering cells $n$ and $n+1$ ) is $p_{n-1} p_{n}$. Thus,

$$
p_{2 n+1}=p_{n} p_{n+1}+p_{n-1} p_{n}=p_{n}\left(p_{n+1}+p_{n-1}\right)
$$

is clearly a multiple of $p_{n}$. Moreover, since $p_{n+1}=2 p_{n}+p_{n-1}$, it follows that $p_{n+1}$ and $p_{n-1}$ have the same parity, and therefore their sum is an even 
integer. Consequently, by Lemma 5 , it is "even" true that $p_{n}$ divides $\frac{1}{2} p_{2 n+1}=$ $\sum_{k=0}^{n} p_{2 k}$.

\subsection{Theorem 3}

Before proving Theorem 3 combinatorially, we first consider a lemma from 3]. Although this lemma is not necessary for our proof of Theorem 3, we include it here for completeness' sake. We will also provide a generalization of it in the final section of this paper. When rephrased in terms of $p_{n}$, it reads as follows:

Lemma 7. For all $n \geq 0$,

$$
p_{n}=\sum_{r \geq 0}\left(\begin{array}{c}
n-r \\
r
\end{array}\right) 2^{n-2 r} .
$$

Proof. The left-hand side of this equality counts all tilings of length $n$. We now consider how many of these tilings contain exactly $r$ dominoes. Such a tiling contains $n-2 r$ squares and, therefore, $n-r$ tiles altogether. There are $\left(\begin{array}{c}n-r \\ r\end{array}\right)$ ways to decide which of these $n-r$ tiles are dominoes and then $2^{n-2 r}$ ways to color the squares. Hence there are $\left(\begin{array}{c}n-r \\ r\end{array}\right) 2^{n-2 r}$ tilings with exactly $r$ dominoes. (Note that this summand is zero whenever $r$ exceeds $\frac{n}{2}$.) Summing over all values of $r$ yields the desired result.

We now proceed to Theorem 3, which we combine into a single identity:

Theorem 8. For all $n \geq 2$,

$$
p_{n-2}+p_{n}=\sum_{r \geq 0} \frac{n}{n-r}\left(\begin{array}{c}
n-r \\
r
\end{array}\right) 2^{n-2 r} .
$$

Proof. In order to prove this theorem, we consider special "modified" tilings we call bracelets. A bracelet of length $n$ is a tiling of an $n$-board with an added wrap-around feature whereby the board is bent into a circle so that cells $n$ and 1, the last and the first cells on the board, are joined. (See [1, Chapter 2] for additional discussion on bracelets.) For bracelets, a domino 
is allowed to simultaneously cover cells $n$ and 1 (which we call "covering the clasp"). When a domino covers the clasp of a bracelet, we say the bracelet is out of phase; otherwise, the bracelet is said to be in phase.

We now proceed by showing that both sides of the equality in this theorem count all bracelets of length $n$ that can be created with white squares, black squares, and gray dominoes. First, with an out of phase bracelet, we can remove the domino covering the clasp to produce a (straight) tiling of length $n-2$. Similarly, an in phase bracelet can be "undone" at the clasp to produce a tiling of length $n$. Altogether, there are $p_{n-2}+p_{n}$ such tilings.

Next, we ask how many such tilings exist with exactly $r$ dominoes. Arguing as in the proof of Lemma 7 above, there are $\left(\begin{array}{c}n-r \\ r\end{array}\right) 2^{n-2 r}$ in phase bracelets with $r$ dominoes, and $\left(\begin{array}{c}n-r-1 \\ r-1\end{array}\right) 2^{n-2 r}$ out of phase bracelets with $r$ dominoes. Since $\left(\begin{array}{c}n-r-1 \\ r-1\end{array}\right)=\frac{r}{n-r}\left(\begin{array}{c}n-r \\ r\end{array}\right)$, we know there are $\frac{n}{n-r}\left(\begin{array}{c}n-r \\ r\end{array}\right) 2^{n-2 r}$ bracelets with exactly $r$ dominoes. Summing over $r$ counts all bracelets of length $n$.

\subsection{Theorem 2}

We now wish to prove our reformulation of Theorem 2. Before proving this theorem, it should be noted that the values of $p_{n-1}+p_{n}$ are well-known [4, A001333. They are the numerators of the continued fraction convergents of $\sqrt{2}$. When $n$ is even, these are known as the NSW numbers studied by Newman, Shanks, and Williams [2] approximately twenty-five years ago in connection with the order of certain simple groups. The interested reader should consult Sloane [4, A002315 for more information on these numbers.

Theorem 9. For all $n \geq 0$,

$$
p_{n-1}+p_{n}=\sum_{r \geq 0}\left(\begin{array}{c}
n+1 \\
2 r
\end{array}\right) 2^{r} .
$$

Proof. We prove this result by counting $(n+1)$-boards with white squares, black squares, and gray dominoes, with the restriction that the tilings do not end in a black square. First, by considering the last tile used in such a tiling (either a domino or a white square), the answer to the question is $p_{n-1}+p_{n}$. 
Next, we note that such a tiling can be constructed by first choosing $X$, a subset of $\{1, \ldots, n+1\}$ of even cardinality, say $X=\left\{x_{1}, \ldots, x_{2 r}\right\}$ with $x_{1}<x_{2}<\cdots<x_{2 r}$. From $X$ we create $r$ intervals $\left[x_{2 j-1}, x_{2 j}\right]$ and can then create $2^{r}$ different tilings as follows. Any cell on the $(n+1)$-board that does not belong to an interval is covered by a white square. An interval of $k \geq 2$ cells can be tiled in one of two ways, either as $k-1$ black squares followed by a white square, or as $k-2$ black squares followed by a domino. (See Figure 2 for one of the four possible colored 11-tilings generated by the intervals $[2,5]$ and $[8,10])$

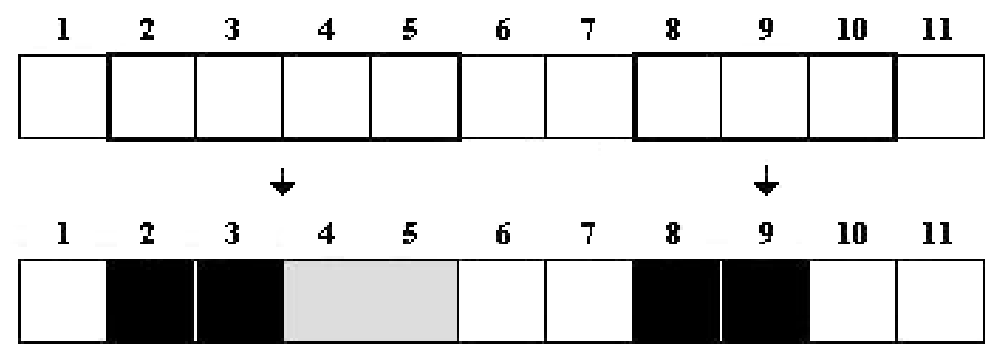

Figure 2: An example of a tiling via intervals

Notice that no interval will end with a black square, and thus the tiling of the $(n+1)$-board, created as the concatenation of all of these smaller tilings, will not end in a black square. Conversely, one can easily show by induction on $n$ that every tiling of a $(n+1)$-board that does not end with a black square has a unique construction in this manner. Summing over $r$ yields the desired result.

\subsection{Theorem 1}

We close this section by considering the following reformulation of Theorem 1. Note that the right-hand side of the equality is the square of an NSW number. 
Theorem 10. For all $n \geq 0$,

$$
\sum_{k=0}^{4 n} p_{k}=\left(p_{2 n-1}+p_{2 n}\right)^{2} .
$$

Proof. The left-hand side counts all tilings of boards of length at most $4 n$ using white squares, black squares, and gray dominoes. Thus we simply need to show that the right-hand side also counts the same tilings.

Note first that the right-hand side clearly counts tiling pairs $(X, Y)$ where $X$ and $Y$ are tilings of $(2 n+1)$-boards that end in a domino or a white square (but not a black square). We now create a bijection from this set to the set of all tilings of length at most $4 n$ by considering the last tile of $X$ and $Y$. Four cases arise:

- Case 1: $X$ and $Y$ both end in a white square. That is, $X=A w$ and $Y=B w$ where $A$ and $B$ are tilings of length $2 n$. Here, $(X, Y)$ is mapped to the tiling $T=A B$ which has length $4 n$ and is breakable at its midpoint.

- Case 2: $X$ ends in a domino, $Y$ ends in a white square. That is, $X=A d$ and $Y=B w$ where $A$ has length $2 n-1$ and $B$ has length $2 n$. Here $(X, Y)$ is mapped to the tiling $A B$, which has length $4 n-1$, and is breakable at its midpoint. (Note that we define the "midpoint" here to be $\left\lfloor\frac{4 n-1}{2}\right\rfloor$ or $2 n-1$.)

- Case 3: $X$ ends in a white square, $Y$ ends in a domino. That is $X=A w$ and $Y=B d$ where $A$ has length $2 n$ and $B$ has length $2 n-1$. (To avoid confusion, we remove the white square at the end of $X$ and the domino at the end of $Y$. Thus $A$ covers cells 1 through $2 n$ and $B$ covers cells 1 through $2 n-1$.) From a visual perspective, we set $A$ above $B$ and look for the last fault line that passes through $A$ and $B$. (A fault line occurs at cell $i$ if both $A$ and $B$ are breakable at cell $i$.) 


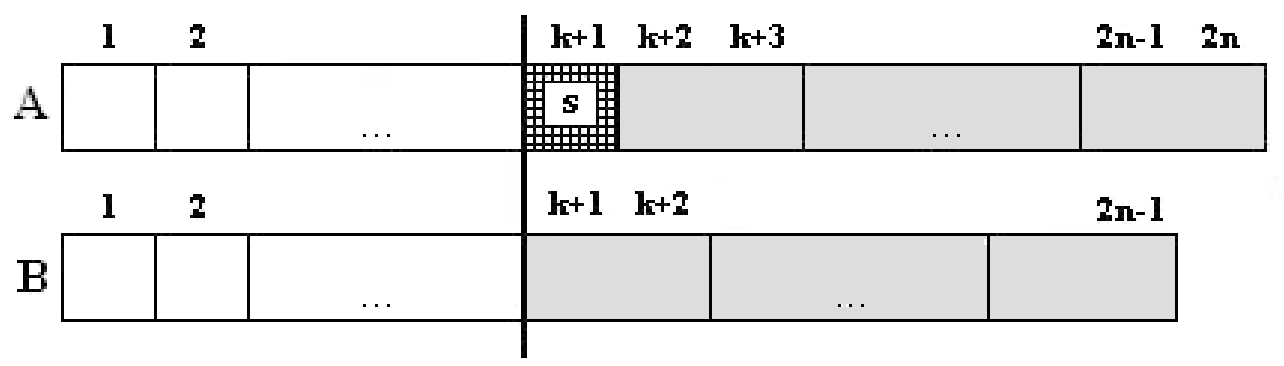

Figure 3: The last fault line occurs at cell $k$.

This fault line occurs at some cell $k$ where $0 \leq k \leq 2 n-1$. (When $k=0$, the only fault line is at "cell 0 ", to the left of cell 1.) To the right of this last fault line, $A$ and $B$ consist entirely of dominoes in staggered formation, except for a single square $s$ on cell $k+1$ in $A$ or $B$ (but not both). In this case, $(X, Y)$ will be mapped to a tiling of even length as follows: Let $C$ denote the subtiling of $A$ covering cells 1 through $k$ and let $D$ denote the subtiling of $B$ covering cells 1 through $k$. If the square $s$ (sitting on cell $k+1$ ) is white, then $(X, Y)$ is mapped to the tiling $C D$, a tiling of length $2 k$ that is breakable at its midpoint. If the square $s$ is black, then $(X, Y)$ is mapped to the tiling $C d D$, a tiling of length $2 k+2$, that is not breakable at its midpoint since a domino starts on cell $k+1$.

Notice that Case 3 covers all of the even length tilings, except for the tilings of length $4 n$ that were covered in Case 1.

It remains to map onto the tilings of odd length at most $4 n-1$, excluding the tilings generated by Case 2 .

- Case 4: $X$ and $Y$ both end in a domino. That is $X=A d$ and $Y=B d$ where $A$ and $B$ have length $2 n-1$. First we "offset" the tilings by shifting $X$ to the right by one cell. (See Figure 4.) $X$ still has length $2 n+1$ but it covers cells 2 through $2 n+2$. Again, 


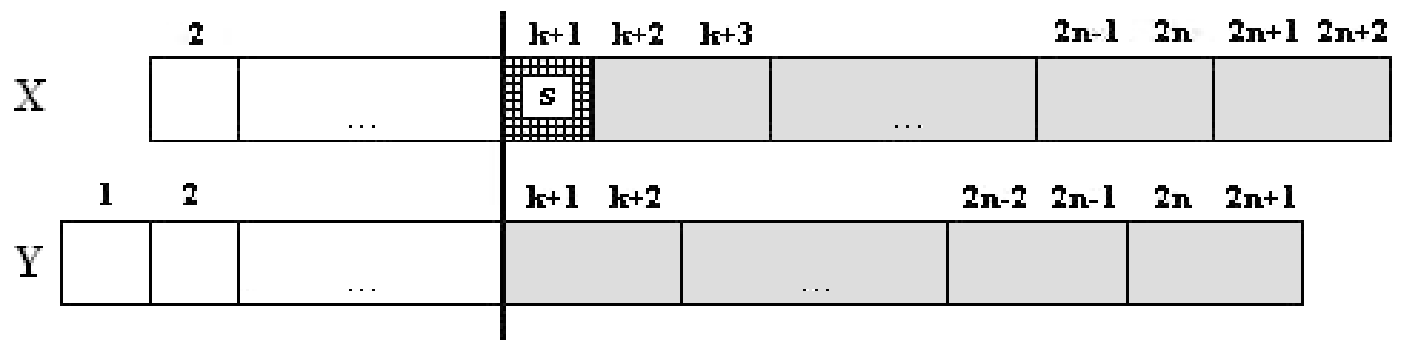

Figure 4: A Case 4 Example

we find the last fault line, which will occur at some cell $k$ where $1 \leq$ $k \leq 2 n-1$. (Note that a fault must exist since $X$ and $Y$ have odd length and must therefore contain at least one square. Also note that $k$ cannot equal $2 n$ or $2 n+1$ since $X$ and $Y$ both end in dominoes.)

As before, to the right of the fault line at cell $k$, we have dominoes in staggered formation, and just a single square $s$ on cell $k+1$ in $A$ or $B$ (but not both). Here $(X, Y)$ will be mapped to a tiling of odd length as follows: Let $C$ denote the subtiling of $X$ covering cells 2 through $k$, and let $D$ denote the subtiling of $Y$ covering cells 1 through $k$. If the square $s$ (sitting on cell $k+1)$ is white, then $(X, Y)$ is mapped to the tiling $C D$, a tiling of length $2 k-1$, that is breakable at its midpoint. If the square $s$ is black, then $(X, Y)$ is mapped to the tiling $C d D$, a tiling of length $2 k+1$, that is not breakable at its midpoint, since a domino starts on cell $k$.

The mapping is easily reversed: Given any tiling of length $j(0 \leq j \leq 4 n)$ we can find its preimage by noting the length and parity of $j$ and whether the tiling is breakable at $\left\lfloor\frac{j}{2}\right\rfloor$. 


\section{Generalizations}

We close by highlighting a number of generalizations of the results above which are obtained almost effortlessly thanks to these tiling proofs. We do not attempt to mention all possible generalizations herein but highlight some of the more straightforward ones that are readily available.

First, we consider a one-parameter generalization of Theorem 9 , Let $p_{n}^{b}$ be defined by $p_{0}^{b}=1, p_{1}^{b}=2$, and $p_{n}^{b}=2 p_{n-1}^{b}+b p_{n-2}^{b}$ for $n \geq 2$ where $b$ is a natural number. By definition, $p_{n}^{1}=p_{n}$ and it is easy to see that $p_{n}^{b}$ counts the number of tilings of an $n$-board with white squares, black squares, and $b$ different colors of dominoes. Then Theorem 9 can be generalized as follows:

Theorem 11. For all $n \geq 0$,

$$
b p_{n-1}^{b}+p_{n}^{b}=\sum_{r \geq 0}\left(\begin{array}{c}
n+1 \\
2 r
\end{array}\right)(b+1)^{r} .
$$

The proof of this generalized theorem closely follows the proof of Theorem 9 given earlier. The only difference is that $b$ choices of colors for the dominoes are available throughout.

To close this section, we consider a two-parameter generalization of $p_{n}$, denoted $p_{n}^{a, b}$ and defined by $p_{0}^{a, b}=1, p_{1}^{a, b}=a$, and $p_{n}^{a, b}=a p_{n-1}^{a, b}+b p_{n-2}^{a, b}$ for $n \geq 2$ where $a$ and $b$ are natural numbers. Thus $p_{n}^{2,1}=p_{n}$ and $p_{n}^{a, b}$ counts the number of tilings of an $n$-board where $a$ different colors of squares and $b$ different colors of dominoes are available. We can then extend our proofs above in the context of these values $p_{n}^{a, b}$ to prove the following generalizations of Lemma 5, the first part of Theorem 6, Lemma 7, and Theorem 8 above as follows:

Lemma 12. For all $n \geq 0$,

$$
p_{2 n+1}^{a, b}=a \sum_{k=0}^{n} b^{n-k} p_{2 k}^{a, b} .
$$

Theorem 13. For all $n \geq 0, p_{n}^{a, b}$ divides $p_{2 n+1}^{a, b}$. 
Lemma 14. For all $n \geq 0$,

$$
p_{n}^{a, b}=\sum_{r \geq 0}\left(\begin{array}{c}
n-r \\
r
\end{array}\right) a^{n-2 r} b^{r} .
$$

Theorem 15. For all $n \geq 0$,

$$
p_{n}^{a, b}+b p_{n-2}^{a, b}=\sum_{r \geq 0} \frac{n}{n-r}\left(\begin{array}{c}
n-r \\
r
\end{array}\right) a^{n-2 r} b^{r} .
$$

\section{References}

[1] A. T. Benjamin and J. J. Quinn, Proofs That Really Count: The Art of Combinatorial Proof, The Dolciani Mathematical Expositions, 27, Mathematical Association of America, Washington, DC, 2003

[2] M. Newman, D. Shanks, and H. C. Williams, Simple groups of square order and an interesting sequence of primes, Acta Arithmetica 38, no. 2 (1980), 129-140

[3] S. F. Santana and J. L. Diaz-Barrero, Some properties of sums involving Pell numbers, Missouri Journal of Mathematical Sciences 18, no. 1 (2006), 33-40

[4] N. J. A. Sloane, The On-Line Encyclopedia of Integer Sequences, published electronically at http://www.research.att.com/ njas/ sequences/, 2006 